\title{
Effect of Platinum-Based Chemotherapy on PD-L1 Expression on Tumor Cells in Non-small Cell Lung Cancer
}

\author{
Junghoon Shin, MD \\ Jin-Haeng Chung, MD, PhD² \\ Se Hyun Kim, MD, PhD ${ }^{1}$ \\ Kyu Sang Lee, MD2 \\ Koung Jin Suh, MD \\ Ji Yun Lee, MD \\ Ji-Won Kim, MD ${ }^{1}$ \\ Jeong-Ok Lee, MD, $P h D^{1}$ \\ Jin-Won Kim, MD, PhD' \\ Yu-Jung Kim, MD, PhD ${ }^{1}$ \\ Keun-Wook Lee, MD, PhD \\ Jee Hyun Kim, MD, PhD' \\ Soo-Mee Bang, MD, PhD' \\ Jong-Seok Lee, MD, PhD ${ }^{1}$
}

${ }^{1}$ Division of Hematology-Oncology, Department of Internal Medicine,

${ }^{2}$ Department of Pathology,

Seoul National University Bundang Hospital, Seoul National University College of

Medicine, Seongnam, Korea

\section{Purpose}

Programmed death-1 (PD-1)/PD-1 ligand (PD-L1) axis blockades have revolutionized the treatment of advanced non-small cell lung cancer (NSCLC). We assessed the effect of platinum-based chemotherapy on tumor PD-L1 expression and its clinical implications.

\section{Materials and Methods}

We used immunohistochemistry to retrospectively evaluate the percentage of tumor cells with membranous PD-L1 staining (tumor proportion score) in paired tumor specimens obtained before and after platinum-based neoadjuvant chemotherapy (NACT) in 86 patients with NSCLC. We analyzed the correlation between the change in PD-L1 tumor proportion score and clinicopathologic characteristics, response to NACT, and survival.

\section{Results}

The PD-L1 tumor proportion score increased in a significant proportion of patients with NSCLC after platinum-based NACT (Wilcoxon signed-rank test, $p=0.002$ ). That pattern was consistent across clinically defined subgroups except for patients with partial response to NACT. Tumors from 26 patients (30.2\%) were PD-L1-negative before NACT but PD-L1-positive after NACT, whereas the reverse pattern occurred in six patients (7\%) (McNemar's test, $p<0.001$ ). Increase in PD-L1 tumor proportion score was significantly associated with lack of response to NACT (Fisher exact test, $p=0.015$ ). There was a tendency, albeit not statistically significant, for patients with an increase in PD-L1 tumor proportion score to have shorter survival.

\section{Conclusion}

Tumor PD-L1 expression increased after platinum-based NACT in a significant proportion of patients with NSCLC. Increase in tumor PD-L1 expression may predict poor clinical outcome.
Correspondence: Se Hyun Kim, MD, PhD Division of Hematology-Oncology, Department of Internal Medicine, Seoul National University Bundang Hospital, Seoul National University College of Medicine, 82 Gumi-ro, Bundang-gu, Seongnam 13620, Korea

Tel: $82-31-787-7071$

Fax: 82-31-787-4098

E-mail: sehyunkim@snubh.org

Received September 27, 2018

Accepted November 2, 2018

Published Online November 5, 2018

\section{Key words}

Non-small cell lung carcinoma, PD-L1, Platinum, Neoadjuvant therapy, Prognosis 


\section{Introduction}

Immune checkpoint inhibitors targeting the programmed death-1 (PD-1)/ PD-1 ligand (PD-L1) axis, such as pembrolizumab, nivolumab, and atezolizumab, have revolutionized the treatment of advanced non-small cell lung cancer (NSCLC). Anti-PD-1 or anti-PD-L1 antibodies have improved the survival of patients with untreated advanced NSCLC or with NSCLC after the failure of platinum-doublet chemotherapy [1-6]. Around $20 \%$ of previously treated patients with NSCLC respond to anti-PD-1 or anti-PD-L1 treatment with a median response duration of 16-20 months [3-6]. Despite long-term benefits in a subset of patients, the majority of unselected patients with NSCLC fail to respond to immune checkpoint inhibitors. PD-L1 expression on tumor cells assessed by immunohistochemistry is the most commonly used predictor of response to PD-1/PD-L1 axis inhibitors and is approved as the only companion diagnostic test for use with pembrolizumab therapy [7]. The tumor PD-L1 expression level can be dynamic over the course of treatment; however, and its predictive value is therefore limited [8-13].

Surgical resection either following (neoadjuvant) or followed by (adjuvant) platinum-based cytotoxic chemotherapy is currently the standard of care in operable, early-stage or locally advanced NSCLC [14]. Platinum agents such as cisplatin and carboplatin are the most active cytotoxic drugs used to treat NSCLC and make up an essential component of chemotherapeutic regimens both in the neoadjuvant or adjuvant setting and in the palliative setting [14]. They mainly exert antitumor activity via the formation of platinum-DNA adducts, which induces a DNA damage-recognition response and cancer cell apoptosis [15]. Preclinical evidence has provided novel insights into their mechanism of action and suggests that modulation of the immune response plays a crucial role in their tumor-killing effect [16]. Importantly, platinum-mediated immunogenic cell death involves the downregulation of both PD-L1 and PD-L2 in dendritic cells and PD-L2 in tumor cells, which results in enhanced tumor recognition by T cells [17]. Clinical trials have shown that the immunogenic effect of platinum-based therapy can be enhanced by combination with PD-1/PD-L1 axis inhibitors $[2,18,19]$.

Few studies have investigated the optimal use of immunotherapy in patients with NSCLC who undergo surgical resection. In order to incorporate immunotherapy into the treatment of those patients, it is crucial to understand the precise impact of platinum-based neoadjuvant chemotherapy (NACT) on tumor PD-L1 expression. Little is known about the prognostic value of PD-L1 upregulation or downregulation following platinum-based chemotherapy in NSCLC. $\mathrm{We}$, therefore, carried out a retrospective study of patients with NSCLC who were treated with platinum-based NACT and subsequent curative lung resection to evaluate the change in tumor PD-L1 expression patterns. We also investigated the relationships between changes in tumor PD-L1

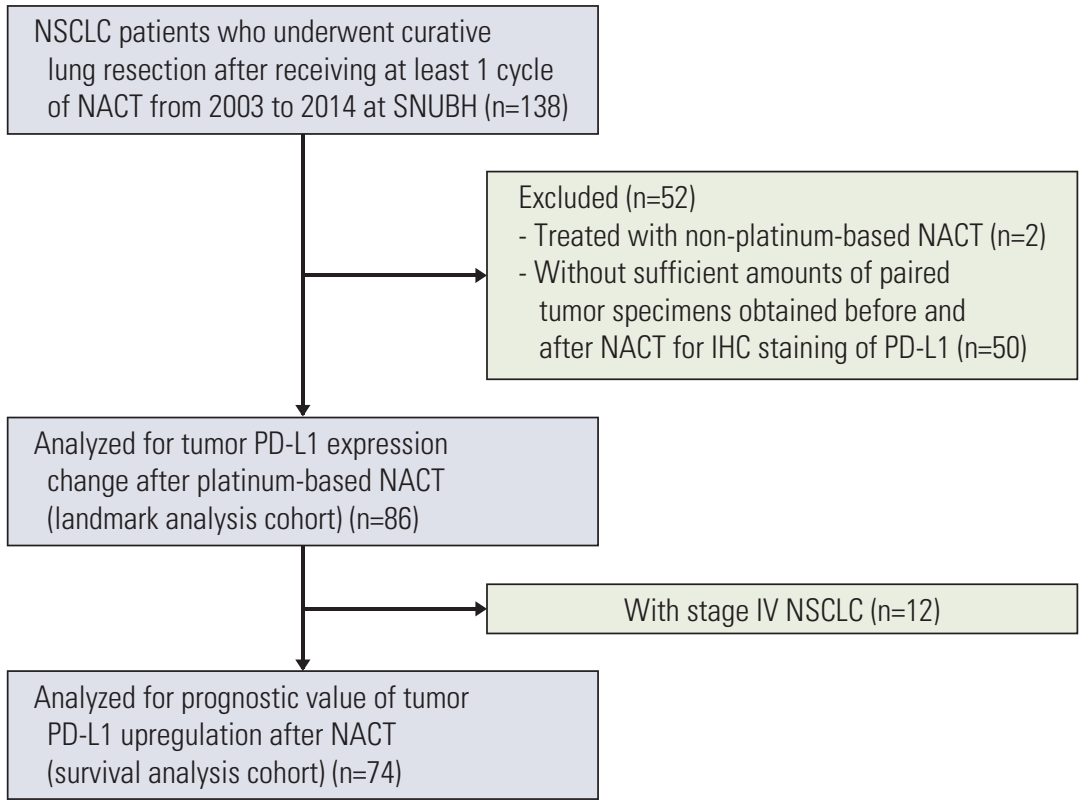

Fig. 1. Flow diagram of patient selection. NSCLC, non-small cell lung cancer; NACT, neoadjuvant chemotherapy; SNUBH, Seoul National University Bundang Hospital; IHC, immunohistochemistry; PD-L1, programmed death ligand 1. 
expression upon NACT and response to chemotherapy and survival.

\section{Materials and Methods}

\section{Patient eligibility and data collection}

We assessed alteration of tumor PD-L1 expression following platinum-based NACT in patients fulfilling the following eligibility criteria (the landmark analysis cohort): (1) had pathologically confirmed NSCLC, (2) underwent curative lung resection after at least one cycle of platinum-based NACT between 2003 and 2014 at Seoul National University Bundang Hospital (SNUBH), and (3) had paired tumor specimens obtained before and after NACT that were adequate for PD-L1 immunostaining (Fig. 1). We analyzed the prognostic value of PD-L1 upregulation after platinum-based NACT only in the subset of patients with stage I-III disease (the survival analysis cohort). Patients with stage IV NSCLC with limited distant metastasis (e.g., solitary brain metastasis) who underwent curative surgery after platinum-based NACT were eligible for the landmark analysis cohort but not for the survival analysis cohort.

We obtained data regarding clinicopathologic characteristics, neoadjuvant treatment, and outcomes from the patients' medical records. We determined the stage of disease according to the seventh edition of the American Joint Committee on Cancer staging guideline after reviewing the patients' clinical records, radiographic images and reports, and pathology reports [20]. We determined dates of death either from medical records or through direct contact with the patients or their family.

\section{Immunohistochemical staining for PD-L1}

We performed immunostaining with antibodies targeting PD-L1 (E1L3N, 1:50, Cell Signaling Technology, Danvers, MA) on tissue slides using the Ventana BenchMark XT autostainer (Ventana Medical Systems, Tucson, AZ) with the ultraView Universal DAB kit (Ventana Medical Systems) according to the manufacturer's standard recommendations. Experienced pathologists (J.H.C. and K.S.L.) determined the percentage of tumor cells with membranous PD-L1 staining of any intensity (tumor proportion score) and grouped the scores into four categories based on cutoffs used in previous studies $(0,<1 \% ; 1,1 \%-5 \% ; 2,5 \%-50 \%$; and $3, \geq 50 \%)[6,21,22]$.

\section{Statistical analysis}

We analyzed categorical variables using either chi-square tests or Fisher exact tests, as appropriate. We used Student's $\mathrm{t}$ tests to compare continuous variables. We analyzed the change in the distribution of PD-L1 tumor proportion scores following NACT using the Wilcoxon signed-rank test. We assessed the change in the proportion of patients that were nominally positive for tumor PD-L1 expression by $\mathrm{McNe}$ mar's test, using a tumor proportion score of $1 \%$ as the cutoff for tumor PD-L1 positivity. We defined disease-free survival (DFS) as the time from surgery to recurrence or death from any cause, whichever came first. We defined overall survival (OS) as the interval from surgery to death from any cause. For estimation of DFS, we censored patients who were disease-free and alive or lost to follow-up at the time of the last assessment of disease status. For estimation of OS, we censored patients who were alive or lost to follow-up on the date of the last follow-up. We calculated DFS and OS using the Kaplan-Meier method and tested for between-group differences using log-rank tests. We tested if PD-L1 upregulation following NACT had an independent prognostic value using Cox proportional hazards regression models adjusted for combinations of patient age at the time of surgery, pretreatment clinical stage, pathologic N category, and pre-NACT or post-NACT tumor proportion scores.

All tests were two-tailed. We considered p-values $<0.05$ to be statistically significant. We performed all statistical analyses using R software, ver. 3.5.0 (R Foundation for Statistical Computing, Vienna, Austria).

\section{Ethical statement}

The institutional review board at SNUBH reviewed and approved the study protocol (approval number: B-1612-375301). Patient consent was waived because of the retrospective nature of the study and lack of patient interaction. All study procedures including data collection and analyses were carried out in accordance with the ethical standards of the Helsinki declaration (revised 2013; World Medical Association).

\section{Results}

\section{Patient characteristics}

A total of 86 patients met the criteria for the landmark analysis cohort (Fig. 1). Among those, 12 patients had stage IV NSCLC and were therefore excluded from the survival 
Table 1. Patient characteristics

\begin{tabular}{|c|c|c|}
\hline Characteristic & Landmark analysis cohort ( $\mathrm{n=86})$ & Survival analysis cohort $(\mathrm{n}=74)$ \\
\hline Age at surgery (yr) & $62(37-79)$ & $62(37-79)$ \\
\hline \multicolumn{3}{|l|}{ Sex } \\
\hline Male & $70(81.4)$ & $63(85.1)$ \\
\hline Female & $16(18.6)$ & $11(14.9)$ \\
\hline \multicolumn{3}{|l|}{ Smoking history } \\
\hline Never-smoker & $11(12.8)$ & $7(9.5)$ \\
\hline Current or former smoker & $75(87.2)$ & $67(90.5)$ \\
\hline \multicolumn{3}{|l|}{ Histologic diagnosis } \\
\hline Adenocarcinoma & $27(31.4)$ & $17(23.0)$ \\
\hline Squamous cell carcinoma & $53(61.6)$ & $51(68.9)$ \\
\hline Others $^{\text {a) }}$ & $6(7.0)$ & $6(8.1)$ \\
\hline \multicolumn{3}{|l|}{ Pretreatment clinical stage } \\
\hline I-II & $22(25.6)$ & $22(29.7)$ \\
\hline IIIA & $40(46.5)$ & $40(54.1)$ \\
\hline IIIB & $12(14.0)$ & $12(16.2)$ \\
\hline IV & $12(14.0)^{\mathrm{b})}$ & 0 \\
\hline \multicolumn{3}{|l|}{ Pathologic T category } \\
\hline урT1-2 & $57(66.3)$ & $48(64.9)$ \\
\hline урТ3-4 & $29(33.7)$ & $26(35.1)$ \\
\hline \multicolumn{3}{|l|}{ Pathologic $\mathbf{N}$ category } \\
\hline ypNo & $24(27.9)$ & $23(31.1)$ \\
\hline ypN1-3 & $62(72.1)$ & $51(68.9)$ \\
\hline \multicolumn{3}{|l|}{ Neoadjuvant treatment category } \\
\hline NACT only & $81(94.2)$ & $70(94.6)$ \\
\hline Chemoradiation (sequential or concurrent) & $5(5.8)$ & $4(5.4)$ \\
\hline \multicolumn{3}{|l|}{ NACT regimen } \\
\hline Gemcitabine plus platinum & $58(67.4)$ & $52(70.3)$ \\
\hline Taxane plus platinum & $24(27.9)$ & $21(28.4)$ \\
\hline Others $^{\mathrm{c})}$ & $4(4.7)$ & $1(1.4)$ \\
\hline \multicolumn{3}{|l|}{ Best objective response to NACT } \\
\hline PR & $45(52.3)$ & $42(56.8)$ \\
\hline SD & $41(47.7)$ & $32(43.2)$ \\
\hline
\end{tabular}

Values are presented as median (range) or number (\%). NACT, neoadjuvant chemotherapy; PR, partial response; SD, stable disease. ${ }^{a}$ Other histologic subtypes include large cell carcinoma $(n=3)$, adenosquamous carcinoma $(n=1)$, pleomorphic car-

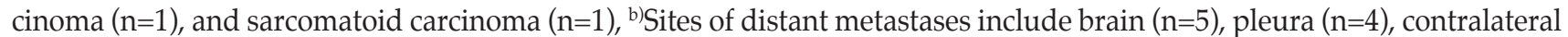
lung $(n=2)$, and rib $(n=1)$, c)Other NACT regimens include pemetrexed plus platinum $(n=2)$, etoposide plus platinum ( $n=1)$, and unknown regimen $(n=1)$ in the landmark analysis cohort, and etoposide plus platinum $(n=1)$ in the survival analysis cohort.

analysis cohort. The patient characteristics for each cohort are summarized in Table 1. A majority of patients were male and current or former smokers, had squamous cell carcinoma, and received at least two cycles of platinum-based NACT; only three $(3.5 \%)$ and two $(2.7 \%)$ patients received one cycle of NACT in the landmark analysis cohort and the survival analysis cohort, respectively. Cisplatin was used in 78 patients $(90.7 \%)$ in the landmark analysis cohort and 67 patients $(90.5 \%)$ in the survival analysis cohort, while the remaining patients received carboplatin in combination with paclitaxel. Neoadjuvant radiotherapy was combined either sequentially or concurrently with NACT in less than $10 \%$ of the patients in both cohorts. Approximately half of the patients in both cohorts achieved partial response (PR) to NACT according to the Response Evaluation Criteria in Solid Tumors, ver. 1.1 [23]. 


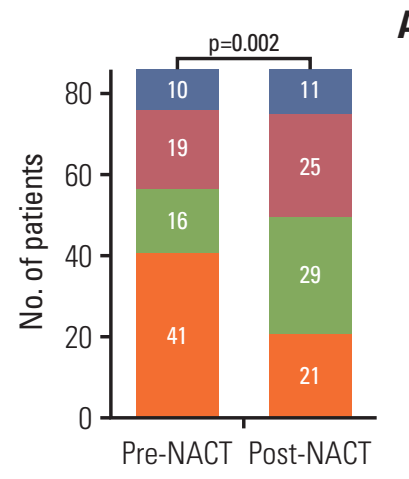

A

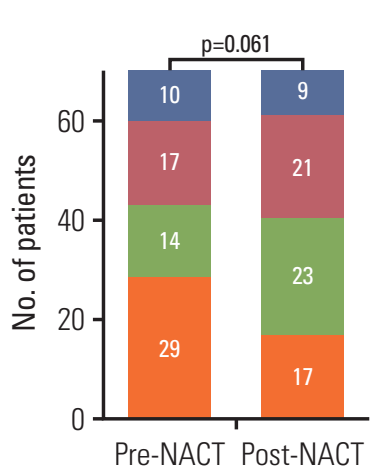

B
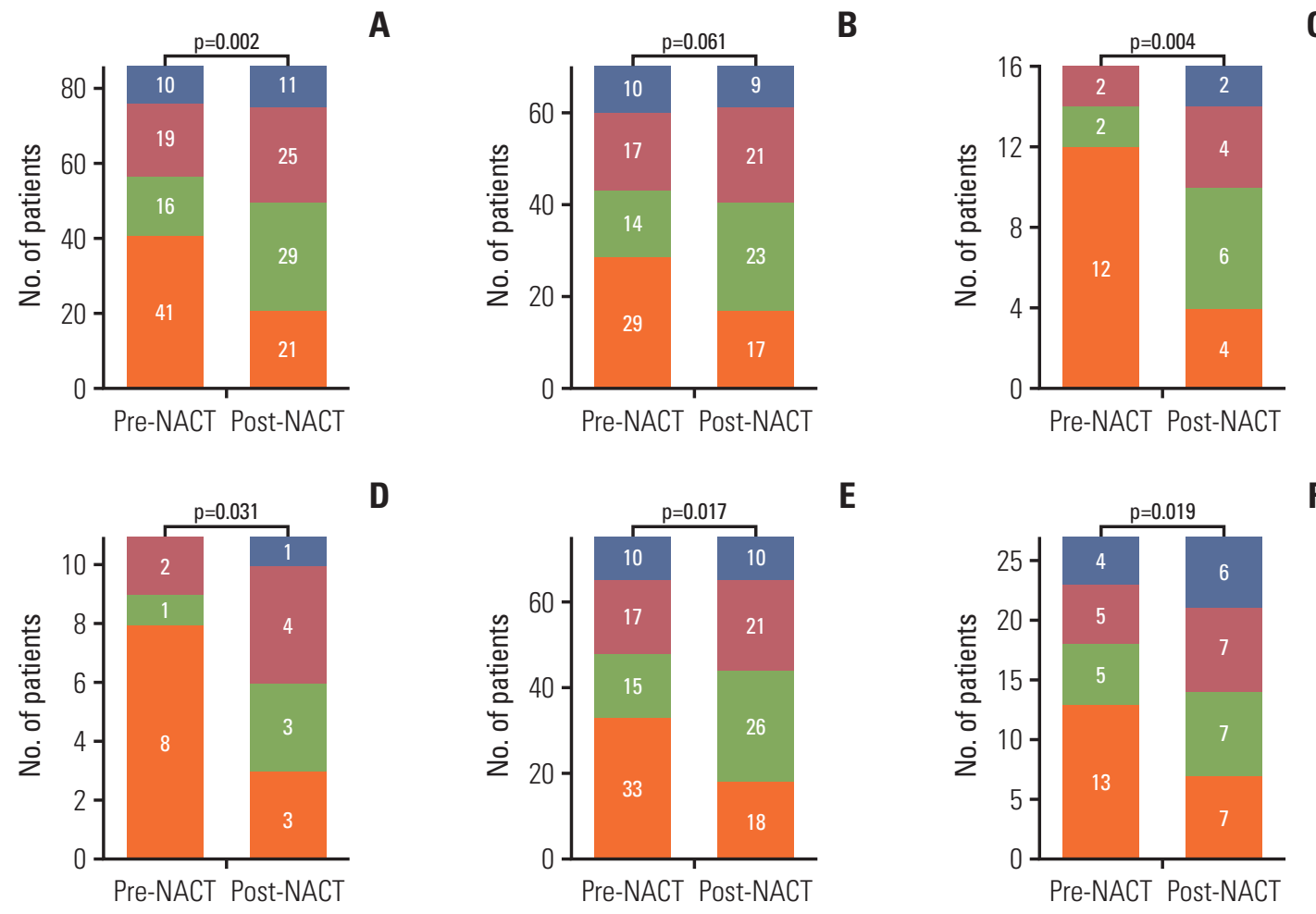

D

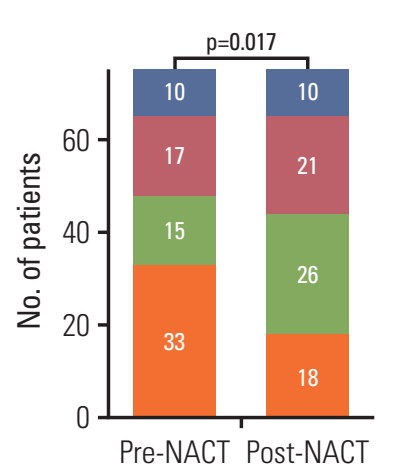

E
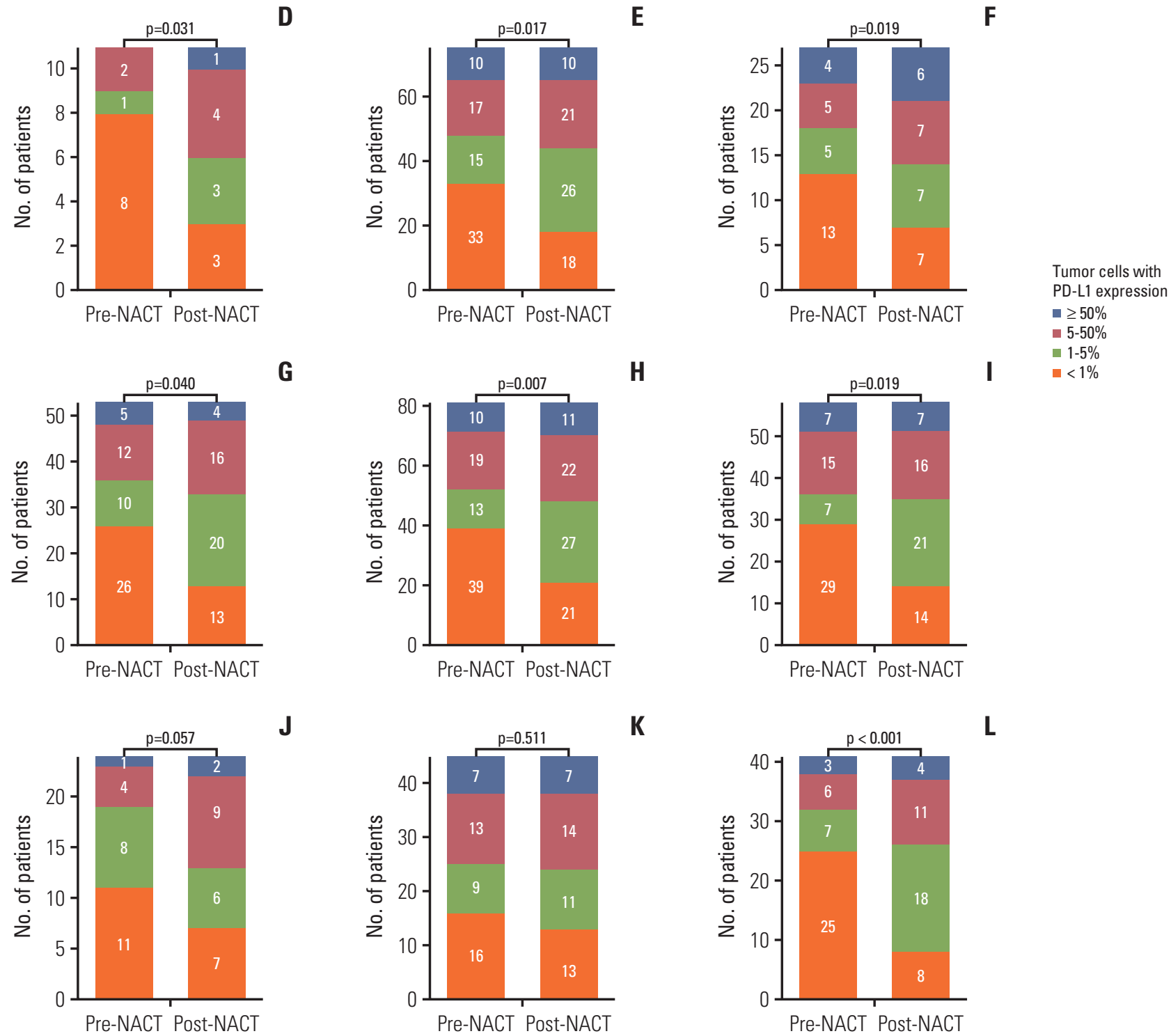

Fig. 2. Distribution of programmed death ligand 1 (PD-L1) tumor proportion score before and after neoadjuvant chemotherapy (NACT) in the landmark analysis cohort (A). Subgroup analyses of males (B), females (C), never-smokers (D), current or former smokers $(\mathrm{E})$, adenocarcinoma $(\mathrm{F})$, squamous cell carcinoma $(\mathrm{G})$, patients who received NACT without radiotherapy $(\mathrm{H})$, gemcitabine-platinum $(\mathrm{I})$, taxane-platinum $(\mathrm{J})$, patients with partial response to NACT $(\mathrm{K})$, and patients with stable disease (L). Numbers in bar plots indicate the number of patients included in each PD-L1 tumor proportion score category. 


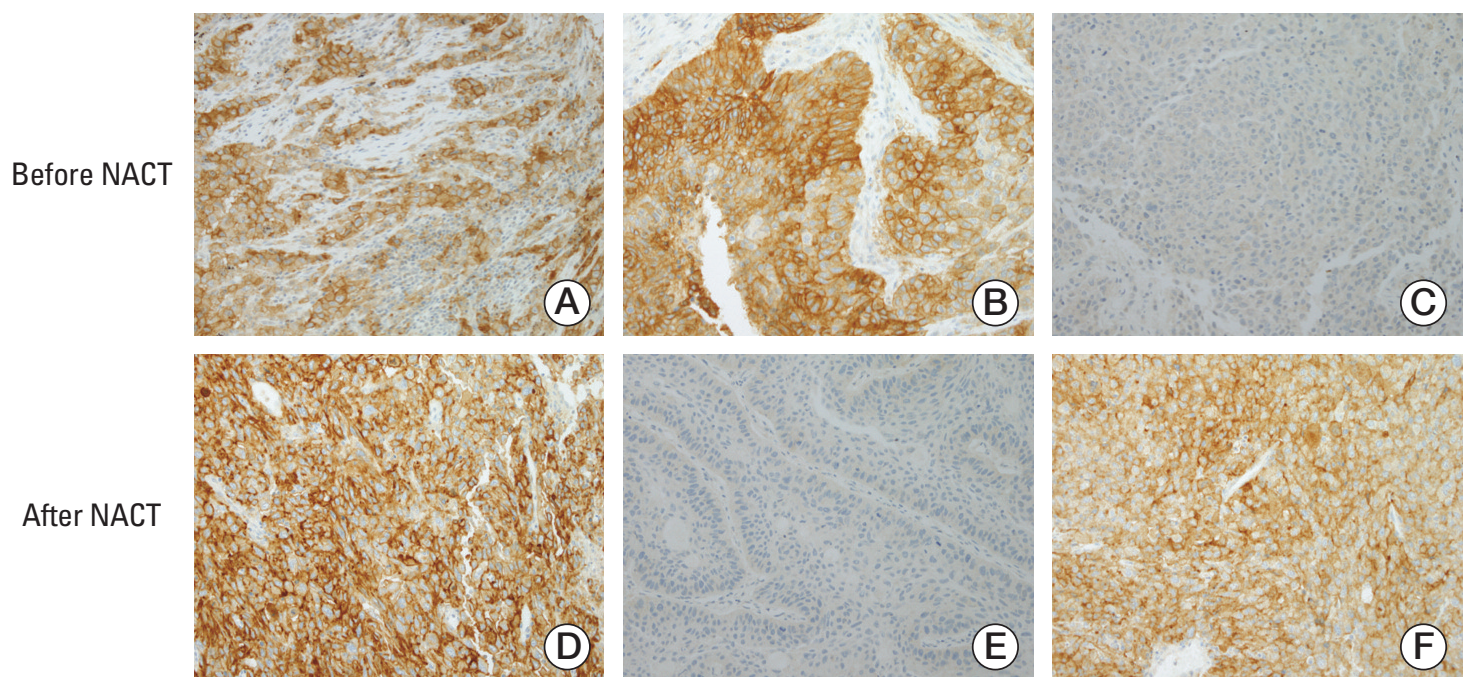

Fig. 3. Representative immunohistochemical staining patterns of programmed death ligand 1 (PD-L1) in tumor specimens. (A, D) PD-L1 tumor proportion score $\geq 50 \%$ before (A) and after (D) neoadjuvant chemotherapy (NACT). (B, E) PD-L1 tumor proportion score $\geq 50 \%$ before (B) and $<1 \%$ after (E) NACT. (C, F) PD-L1 tumor proportion score $<1 \%$ before (C) and $\geq 50 \%$ after (F) NACT (A-F, ×200).

\section{Change in tumor PD-L1 expression following platinum- based NACT}

Before treatment, $41(47.7 \%), 16(18.6 \%), 19(22.1 \%)$, and 10 $(11.6 \%)$ patients were in the PD-L1 tumor proportion score ranges of $<1 \%, 1 \%-5 \%, 5 \%-50 \%$, and $\geq 50 \%$, respectively, in the landmark analysis cohort. The overall proportion of patients with positive tumor PD-L1 expression increased significantly after treatment with platinum-based NACT (45/86 [52.3\%] before NACT vs. 65/86 [75.6\%] after NACT; $p=0.002$ ) (Fig. 2A). That trend was consistent across subgroups stratified by sex, smoking history, histologic subtype, neoadjuvant treatment category, NACT regimen, and response to NACT (Fig. 2B-L), except for the subgroup of patients that achieved PR to NACT (Fig. 2K). The distributions of pre-NACT and post-NACT PD-L1 tumor proportion scores differed significantly within most subgroups, although they were only marginally different among males (Fig. 2B) and patients treated with taxane-platinum regimens (Fig. 2J). Notably, 26 patients were negative for tumor PD-L1 expression (tumor proportion score $<1 \%$ ) before NACT and positive for tumor PD-L1 expression (tumor proportion score $\geq 1 \%$ ) after NACT, whereas only six patients $(7 \%)$ exhibited the reverse pattern $(\mathrm{p}<0.001)$. Overall, $34(39.5 \%), 12(14 \%)$, and $40(46.5 \%)$ patients showed an increased, decreased, or unchanged PD-L1 tumor proportion score following NACT, respectively (Fig. 3).

To assess the clinicopathologic characteristics associated with PD-L1 upregulation following NACT, we divided the patients into two groups based on the change in PD-L1 tumor proportion score following NACT. The 'upward' group included patients whose PD-L1 tumor proportion score increased after NACT. The 'unchanged or downward' group included the remaining patients. Comparison of the two groups indicated that the response to NACT was significantly correlated with the direction of change in PD-L1 tumor proportion score $(\mathrm{p}=0.015)$; patients who did not respond to NACT were twice as likely to show an upward change in PD-L1 tumor proportion score than those who achieved PR (Table 2). That result is in line with the results in Fig. $2 \mathrm{~K}$ and $\mathrm{L}$, which show that the proportion of tumor cells expressing PD-L1 increased after NACT only in tumors from patients who did not respond to NACT.

\section{Prognostic impact of tumor PD-L1 upregulation follow- ing platinum-based NACT}

During a median follow-up of 7.3 years in the survival analysis cohort, 47 patients (63.5\%) experienced disease recurrence and 41 patients $(55.4 \%)$ died. DFS did not differ significantly between patients with increased PD-L1 tumor proportion score following NACT and those with unchanged or decreased PD-L1 tumor proportion score following NACT (median DFS, 11.8 months vs. 12 months) (Fig. 4A). Similarly, OS also did not differ significantly between the two groups (median OS, 3.8 years vs. 7.7 years) (Fig. 4B). Those results were consistent with multivariable Cox models adjusted for predefined sets of variables (Table 3). After adjustment, 
Table 2. Direction of change in PD-L1 tumor proportion score following NACT in relation to clinicopathologic characteristics

\begin{tabular}{|c|c|c|c|}
\hline Clinicopathologic variable & $\begin{array}{l}\text { Unchanged or downward } \\
\qquad(\mathrm{n}=52)\end{array}$ & $\begin{array}{l}\text { Upward } \\
(\mathrm{n}=34)\end{array}$ & p-value \\
\hline Age at surgery (yr) & $62.5(37-76)$ & $61(38-79)$ & 0.572 \\
\hline \multicolumn{4}{|l|}{ Sex } \\
\hline Male & $46(88.5)$ & $24(70.6)$ & 0.049 \\
\hline Female & $6(11.5)$ & $10(29.4)$ & \\
\hline \multicolumn{4}{|l|}{ Smoking history } \\
\hline Never-smoker & $5(9.6)$ & $6(17.6)$ & 0.331 \\
\hline Current or former smoker & $47(90.4)$ & $28(82.4)$ & \\
\hline \multicolumn{4}{|l|}{ Histological diagnosis } \\
\hline Adenocarcinoma & $17(32.7)$ & $10(29.4)$ & 0.476 \\
\hline Squamous cell carcinoma & $30(57.7)$ & $23(67.6)$ & \\
\hline Others & $5(9.6)$ & $1(2.9)$ & \\
\hline \multicolumn{4}{|l|}{ Pretreatment clinical stage } \\
\hline I-II & $11(21.2)$ & $11(32.4)$ & 0.314 \\
\hline III-IV & $41(78.8)$ & $23(67.6)$ & \\
\hline \multicolumn{4}{|l|}{ Pathologic T category } \\
\hline урТ1-2 & $36(69.2)$ & $21(61.8)$ & 0.493 \\
\hline урТ3-4 & $16(30.8)$ & $13(38.2)$ & \\
\hline \multicolumn{4}{|l|}{ Pathologic $\mathbf{N}$ category } \\
\hline ypNo & $14(26.9)$ & $10(29.4)$ & 0.811 \\
\hline ypN1-3 & $38(73.1)$ & $24(70.6)$ & \\
\hline \multicolumn{4}{|l|}{ NACT regimen } \\
\hline Gemcitabine plus platinum & $36(69.2)$ & $22(64.7)$ & 0.644 \\
\hline Taxane plus platinum & $13(25.0)$ & $11(32.4)$ & \\
\hline Others & $3(5.8)$ & $1(2.9)$ & \\
\hline \multicolumn{4}{|c|}{ Best objective response to NACT } \\
\hline PR & $33(63.5)$ & $12(35.3)$ & 0.015 \\
\hline SD & $19(36.5)$ & $22(64.7)$ & \\
\hline
\end{tabular}

Values are presented as median (range) or number (\%). PD-L1, programmed death ligand 1; NACT, neoadjuvant chemotherapy; PR, partial response; SD, stable disease.

PD-L1 upregulation following NACT was not significantly associated with increased or decreased DFS and OS; however, visual inspection of the survival curves and the results of the multivariable Cox models indicated a trend toward poorer OS in patients whose PD-L1 tumor proportion score increased. That tendency was consistent across subgroups defined by sex, smoking history, histologic diagnosis, pretreatment clinical stage, pathologic $\mathrm{T}$ category assessed at surgery, NACT regimen, and response to NACT. The hazard ratio for death was less than one only in patients who received taxane-platinum combination therapy (Fig. 5).

\section{Discussion}

We found that the PD-L1 tumor proportion score increased in a significant proportion of patients with NSCLC after treatment with platinum-based NACT. That pattern of change in tumor PD-L1 expression was consistent across clinically defined subgroups, except for the patients who achieved PR to NACT. Almost one-third of all the patients were negative for PD-L1 expression before NACT but positive for PD-L1 expression after NACT, based on a cutoff PD-L1 tumor proportion score of $1 \%$, which is currently approved as an indication for pembrolizumab therapy after the failure of platinum-based doublet chemotherapy $[3,14]$. That result implies that the tumor PD-L1 expression level should be reassessed after platinum-based chemotherapy for appropriate selection 
A

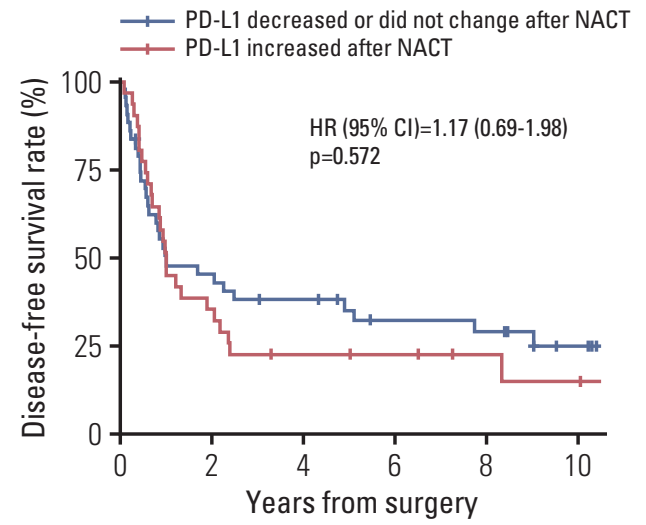

No. at risk

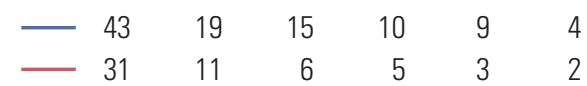

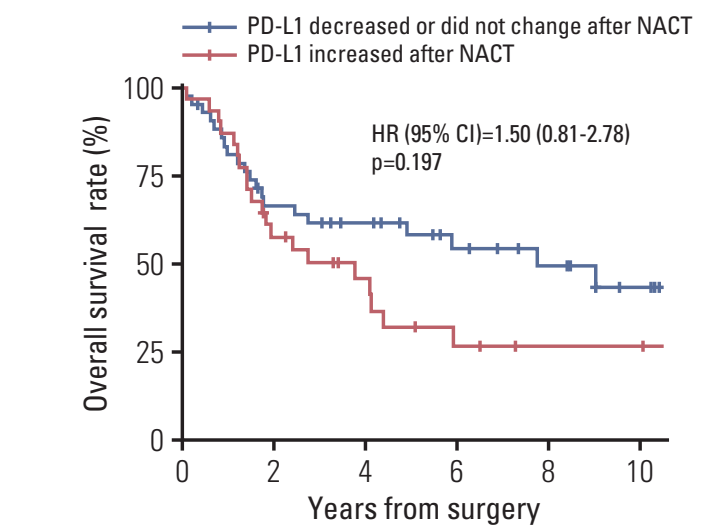

No. at risk

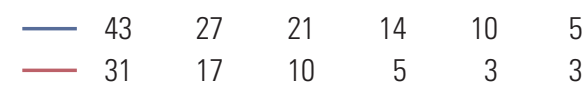

Fig. 4. Disease-free survival (A) and overall survival (B) according to the direction of change in programmed death ligand 1 (PD-L1) tumor proportion score following platinum-based neoadjuvant chemotherapy (NACT). HR, hazard ratio; CI, confidence interval.

Table 3. Prognostic impact of PD-L1 upregulation (versus no change or downregulation) following platinum-based NACT

\begin{tabular}{|c|c|c|c|c|}
\hline \multirow{2}{*}{ Model } & \multicolumn{2}{|c|}{ Disease-free survival } & \multicolumn{2}{|c|}{ Overall survival } \\
\hline & HR $(95 \% \mathrm{CI})$ & p-value & HR $(95 \%$ CI) & p-value \\
\hline Model 1 ${ }^{\text {a) }}$ & $1.17(0.67-2.03)$ & 0.578 & $1.69(0.89-3.22)$ & 0.111 \\
\hline Model 2b) & $1.32(0.73-2.39)$ & 0.366 & $1.60(0.80-3.18)$ & 0.182 \\
\hline Model 3c) & $0.89(0.46-1.70)$ & 0.718 & $1.53(0.69-3.38)$ & 0.290 \\
\hline
\end{tabular}

PD-L1, programmed death ligand 1; NACT, neoadjuvant chemotherapy; HR, hazard ratio; CI, confidence interval. ${ }^{\text {al Model }}$ 1 is adjusted for age ( $\geq 65 \mathrm{vs.}<65$ ), pretreatment clinical stage (I-II vs. III), and pathologic N category (ypN0 vs. ypN1 vs. ypN2 vs. ypN3), b)Model 2 is adjusted for age ( $\geq 65$ vs. $<65$ ), pretreatment clinical stage (I-II vs. III), pathologic $\mathrm{N}$ category (ypN0 vs. ypN1 vs. ypN2 vs. ypN3), and pre-NACT PD-L1 tumor proportion score (<1\% vs. 1\%-50\% vs. $\geq 50 \%)$, c)Model 3 is adjusted for age ( $\geq 65$ vs. $<65$ ), pretreatment clinical stage (I-II vs. III), pathologic N category (ypN0 vs. ypN1 vs. ypN2 vs. ypN3), and post-NACT PD-L1 tumor proportion score (<1\% vs. 1-50\% vs. $\geq 50 \%)$.

of immunotherapy candidates. It also may support the design of clinical trials that incorporate PD-1/PD-L1 axis inhibitors into the treatment of patients with early-stage NSCLC.

A few retrospective studies recently investigated the alteration of tumor PD-L1 expression in patients with NSCLC who received platinum-based NACT (with or without radiotherapy) [9-13]. Those studies assessed much smaller numbers of paired tumor specimens than our study, or they focused only on a specific histologic subtype and therefore produced conflicting results. It is possible that tumor cells with PD-L1 downregulation as a result of exposure to platinum agents might undergo enhanced immune clearance, resulting in enrichment of PD-L1-upregulated tumor cells in post-NACT tumor specimens. Indeed, our findings are consistent with the results of previous studies $[8,11,13]$, although the small cohort size warrants confirmation in a larger cohort.

The predictive or prognostic value of tumor PD-L1 expression is an area of debate [24-28]. In our study, patients with increased PD-L1 tumor proportion score following platinumbased NACT had worse response to chemotherapy and tended to live for a shorter time than patients with unchanged or downregulated tumor PD-L1 expression, although the differences in prognosis were not statistically significant. Similarly, results from previous studies indicated that 


\begin{tabular}{lllll}
\hline Subgroup & HR (95\% CI) & p-value \\
Overall & No. & $1.17(0.69-1.98)$ & 0.573 \\
Age at surgery \\
$<65$
\end{tabular}

Fig. 5. Subgroup analyses of the prognostic value of increased (versus unchanged or decreased) programmed death ligand 1 tumor proportion score following platinum-based neoadjuvant chemotherapy (NACT) for disease-free survival (A) and overall survival (B). Note that subgroups with less than 10 patients are omitted. The vertical dashed line indicates the hazard ratio (HR) for all patients in the survival analysis cohort. CI, confidence interval; PR, partial response; SD, stable disease. (Continued to the next page)

increased tumor PD-L1 expression following NACT was associated with poor response to chemotherapy and poor survival $[9,10,13]$. That, along with the frequent upregulation of tumor PD-L1 expression following platinum-based chemotherapy, suggests that patients with operable, early-stage or locally advanced NSCLC may benefit from postoperative PD-1/ PD-L1 axis-inhibitor therapy after completion of standard neoadjuvant or adjuvant chemotherapy. The ongoing EORTC-ETOP phase 3 trial may confirm the validity of that hypothesis [29].

Our study has limitations. First and most importantly, the small numbers of patients included in the study cohorts lim- ited the statistical power. Although we observed a consistent tendency toward shorter survival in patients with an upward change in tumor PD-L1 expression, the trend did not reach statistical significance. Additionally, the pre-NACT specimens were mostly obtained by percutaneous or transbronchial lung biopsy, whereas the post-NACT specimens were obtained by surgical resection. Therefore, there might be a systematic difference in measured tumor PD-L1 expression caused by a discrepancy in tissue acquisition techniques. Lastly, the study cohorts represented a heterogeneous patient population with various histologic subtypes and clinical stages. For that reason, most of the subgroups included only 


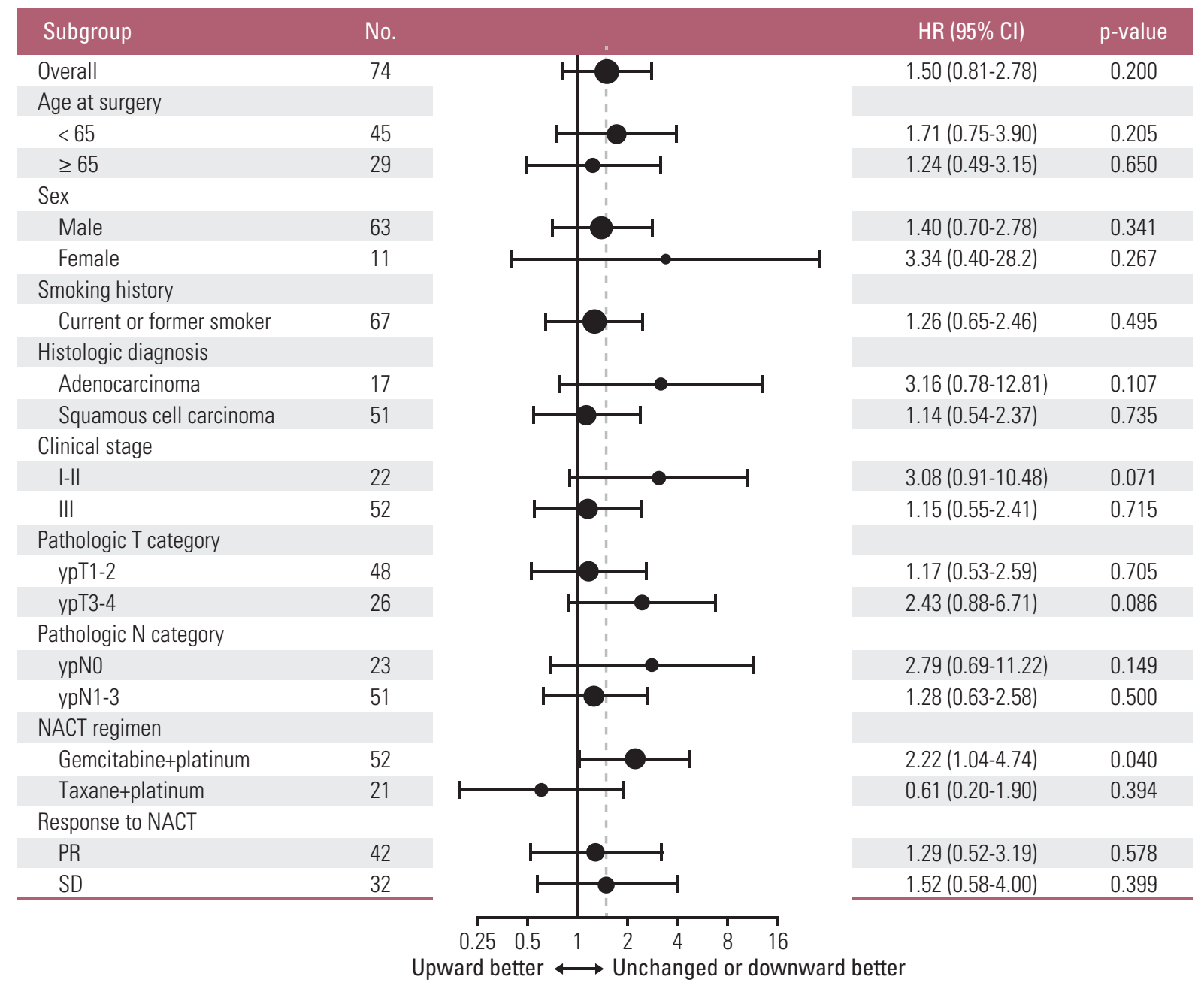

Fig. 5. (Continued from the previous page)

a small number of patients, which made it difficult to draw reliable conclusions from the subgroup analyses.

In summary, using matched pre-NACT and post-NACT tumor specimens obtained from patients with NSCLC, we demonstrated that the proportion of tumor cells expressing PD-L1 tended to increase after platinum-based chemotherapy, regardless of clinical characteristics and pathologic subtypes. The patients whose PD-L1 tumor proportion score increased following NACT tended to have poorer chemotherapy response and OS than patients whose PD-L1 tumor proportion score decreased or was unchanged. Our findings emphasize the need for reassessment of tumor PD-L1 expression after platinum-based chemotherapy and the potential benefit of postoperative PD-1/PD-L1 checkpoint inhibitor treatment for patients with operable NSCLC who undergo platinum-based neoadjuvant or adjuvant chemotherapy.

\section{Conflicts of Interest}

Conflict of interest relevant to this article was not reported.

\section{Acknowledgments}

This study was supported by the grants from the SNUBH Research Fund (grant number: 02-2014-042 to SHK), Korea Health Technology R\&D Project through the Korea Health Industry Development Institute (KHIDI), funded by the Ministry of Health \& Welfare, Republic of Korea (grant number: HI17C1290 to JHC) and the National Research Foundation of Korea (NRF) Grant funded by the Korean Government (MSIT) (No. 2017R1A5A1015626 to JHC). This manuscript has been edited by native English-speaking experts associated with BioScience Writers LLC, Houston, TX, USA. 


\section{References}

1. Reck M, Rodriguez-Abreu D, Robinson AG, Hui R, Csoszi T, Fulop A, et al. Pembrolizumab versus chemotherapy for PD-L1-positive non-small-cell lung cancer. N Engl J Med. 2016;375:1823-33.

2. Gandhi L, Rodriguez-Abreu D, Gadgeel S, Esteban E, Felip E, De Angelis F, et al. Pembrolizumab plus chemotherapy in metastatic non-small-cell lung cancer. N Engl J Med. 2018;378: 2078-92.

3. Herbst RS, Baas P, Kim DW, Felip E, Perez-Gracia JL, Han JY, et al. Pembrolizumab versus docetaxel for previously treated, PD-L1-positive, advanced non-small-cell lung cancer (KEYNOTE-010): a randomised controlled trial. Lancet. 2016;387: 1540-50.

4. Brahmer J, Reckamp KL, Baas P, Crino L, Eberhardt WE, Poddubskaya E, et al. Nivolumab versus docetaxel in advanced squamous-cell non-small-cell lung cancer. N Engl J Med. 2015;373:123-35.

5. Borghaei H, Paz-Ares L, Horn L, Spigel DR, Steins M, Ready $\mathrm{NE}$, et al. Nivolumab versus docetaxel in advanced nonsquamous non-small-cell lung cancer. N Engl J Med. 2015;373:162739.

6. Rittmeyer A, Barlesi F, Waterkamp D, Park K, Ciardiello F, von Pawel J, et al. Atezolizumab versus docetaxel in patients with previously treated non-small-cell lung cancer (OAK): a phase 3, open-label, multicentre randomised controlled trial. Lancet. 2017;389:255-65.

7. Sul J, Blumenthal GM, Jiang X, He K, Keegan P, Pazdur R. FDA approval summary: pembrolizumab for the treatment of patients with metastatic non-small cell lung cancer whose tumors express programmed death-ligand 1. Oncologist. 2016;21:643-50.

8. Parra ER, Villalobos P, Behrens C, Jiang M, Pataer A, Swisher $S G$, et al. Effect of neoadjuvant chemotherapy on the immune microenvironment in non-small cell lung carcinomas as determined by multiplex immunofluorescence and image analysis approaches. J Immunother Cancer. 2018;6:48.

9. Sheng J, Fang W, Yu J, Chen N, Zhan J, Ma Y, et al. Expression of programmed death ligand-1 on tumor cells varies pre and post chemotherapy in non-small cell lung cancer. Sci Rep. 2016;6:20090.

10. Fujimoto D, Uehara K, Sato Y, Sakanoue I, Ito M, Teraoka S, et al. Alteration of PD-L1 expression and its prognostic impact after concurrent chemoradiation therapy in non-small cell lung cancer patients. Sci Rep. 2017;7:11373.

11. Song Z, Yu X, Zhang Y. Altered expression of programmed death-ligand 1 after neo-adjuvant chemotherapy in patients with lung squamous cell carcinoma. Lung Cancer. 2016;99:16671.

12. Rojko L, Reiniger L, Teglasi V, Fabian K, Pipek O, Vagvolgyi A, et al. Chemotherapy treatment is associated with altered PD-L1 expression in lung cancer patients. J Cancer Res Clin Oncol. 2018;144:1219-26.

13. Zhang P, Ma Y, Lv C, Huang M, Li M, Dong B, et al. Upregulation of programmed cell death ligand 1 promotes resistance response in non-small-cell lung cancer patients treated with neo-adjuvant chemotherapy. Cancer Sci. 2016;107:1563-71.

14. Ettinger DS, Wood DE, Aisner DL, Akerley W, Bauman J, Chirieac LR, et al. Non-small cell lung cancer, version 5.2017, NCCN clinical practice guidelines in oncology. J Natl Compr Canc Netw. 2017;15:504-35.

15. Kelland L. The resurgence of platinum-based cancer chemotherapy. Nat Rev Cancer. 2007;7:573-84.

16. Hato SV, Khong A, de Vries IJ, Lesterhuis WJ. Molecular pathways: the immunogenic effects of platinum-based chemotherapeutics. Clin Cancer Res. 2014;20:2831-7.

17. Lesterhuis WJ, Punt CJ, Hato SV, Eleveld-Trancikova D, Jansen BJ, Nierkens $S$, et al. Platinum-based drugs disrupt STAT6-mediated suppression of immune responses against cancer in humans and mice. J Clin Invest. 2011;121:3100-8.

18. Rizvi NA, Hellmann MD, Brahmer JR, Juergens RA, Borghaei $\mathrm{H}$, Gettinger S, et al. Nivolumab in combination with platinum-based doublet chemotherapy for first-line treatment of advanced non-small-cell lung cancer. J Clin Oncol. 2016;34: 2969-79.

19. Liu SV, Camidge DR, Gettinger SN, Giaccone G, Heist RS, Hodi FS, et al. Long-term survival follow-up of atezolizumab in combination with platinum-based doublet chemotherapy in patients with advanced non-small-cell lung cancer. Eur J Cancer. 2018;101:114-22.

20. Goldstraw P, Crowley J, Chansky K, Giroux DJ, Groome PA, Rami-Porta R, et al. The IASLC Lung Cancer Staging Project: proposals for the revision of the TNM stage groupings in the forthcoming (seventh) edition of the TNM Classification of malignant tumours. J Thorac Oncol. 2007;2:706-14.

21. Herbst RS, Soria JC, Kowanetz M, Fine GD, Hamid O, Gordon MS, et al. Predictive correlates of response to the anti-PD-L1 antibody MPDL3280A in cancer patients. Nature. 2014;515: 563-7.

22. Fehrenbacher L, Spira A, Ballinger M, Kowanetz M, Vansteenkiste J, Mazieres J, et al. Atezolizumab versus docetaxel for patients with previously treated non-small-cell lung cancer (POPLAR): a multicentre, open-label, phase 2 randomised controlled trial. Lancet. 2016;387:1837-46.

23. Eisenhauer EA, Therasse P, Bogaerts J, Schwartz LH, Sargent D, Ford R, et al. New response evaluation criteria in solid tumours: revised RECIST guideline (version 1.1). Eur J Cancer. 2009;45:228-47.

24. Tsao MS, Le Teuff G, Shepherd FA, Landais C, Hainaut P, Filipits $\mathrm{M}$, et al. PD-L1 protein expression assessed by immunohistochemistry is neither prognostic nor predictive of benefit from adjuvant chemotherapy in resected non-small cell lung cancer. Ann Oncol. 2017;28:882-9.

25. Sun JM, Zhou W, Choi YL, Choi SJ, Kim SE, Wang Z, et al. Prognostic significance of PD-L1 in patients with non-small cell lung cancer: a large cohort study of surgically resected cases. J Thorac Oncol. 2016;11:1003-11.

26. Wang A, Wang HY, Liu Y, Zhao MC, Zhang HJ, Lu ZY, et al. The prognostic value of PD-L1 expression for non-small cell 
lung cancer patients: a meta-analysis. Eur J Surg Oncol. 2015;41:450-6.

27. Tokito T, Azuma K, Kawahara A, Ishii H, Yamada K, Matsuo $\mathrm{N}$, et al. Predictive relevance of PD-L1 expression combined with CD8+ TIL density in stage III non-small cell lung cancer patients receiving concurrent chemoradiotherapy. Eur J Cancer. 2016;55:7-14.

28. Cooper WA, Tran T, Vilain RE, Madore J, Selinger CI, Kohonen-Corish $\mathrm{M}$, et al. PD-L1 expression is a favorable prognos- tic factor in early stage non-small cell carcinoma. Lung Cancer. 2015;89:181-8.

29. O'Brien ME, Hasan B, Dafni U, Menis J, Peters S, De Waele M, et al. EORTC-ETOP randomized, phase 3 trial with anti-PD-1 monoclonal antibody pembrolizumab versus placebo for patients with early stage non-small cell lung cancer (NSCLC) after resection and standard adjuvant chemotherapy: PEARLS (NCT02504372). J Clin Oncol. 2016;34(15 Suppl):TPS8571. 